\title{
El don de la intuición en la interpretación heideggeriana de Kant
}

\section{The Gift of Intuition in Heidegger’s Interpretation of Kant}

\author{
SUSI FERRARELLO* \\ (Loyola University, “John Felice Rome Center”)
}

\begin{abstract}
Resumen: En el presente artículo nuestra manera de proceder estará marcada por la individuación de los puntos del pensamiento de Kant sobre los que Heidegger ha ejercido su forzamiento. Nos concentraremos en forma particular sobre la primera interpretación de la Crítica kantiana que el filósofo dio en las lecciones del semestre de invierno 1927/28. En efecto, es en este curso donde es posible reconocer la primera intervención por parte de Heidegger dirigida a la superación de la metafísica tradicional kantiana y a la construcción de su propia ontología. Insistiremos de manera particular sobre los conceptos de intuición, imaginación y representación que consideramos fundamentales para la formulación de esa ontología. La descripción de este núcleo de conceptos parece, en efecto, no sólo forzar el sentido que Kant les asignaba, sino que además da lugar a ciertas ambigüedades que repercuten en la definición heideggeriana de ontología. Palabras Clave: ontología, intuición, imaginación, representación
\end{abstract}

\begin{abstract}
In this article, we will focus on the loci of interpretation of Kant's thought in which it seems that Heidegger has forced his interpretation. We will concentrate, in particular, on the first interpretation of the Kantian Criticism on which the philosopher lectured in the winter semester $1927 / 28$. Indeed, it is in these lectures that we can observe Heidegger's first attempt to overcome the traditional Kantian metaphysics in favor of his ontology. We believe, in particular, that the concepts of intuition, imagination, and representation are fundamental for the formulation of that ontology. The description of this core of concepts seems, in effect, not only to force the meaning that Kant assigned to each of them, but also gives rise to certain ambiguities that reverberate in the Heideggerian definition of ontology.
\end{abstract}

Keywords: ontology, intuition, imagination, representation

\section{Introducción}

Los críticos señalan con frecuencia que en Heidegger el abordaje de la tradición se encuentra estrechamente asociado con la transformación ${ }^{1}$. En efecto, para el filósofo, interpretar significa forzar. La interpretación eficaz de un texto no puede limitarse a relatar lo que el mismo texto

\footnotetext{
* Agradezco profundamente a Giusy Liberti, Piero Mari y Federico Jose Arena por el valioso trabajo de traducción que han llevado a cabo.

1 Sobre este punto véase: Gadamer, 1983, p. 24, pp. 138-149; Volpi, 1984; Volpi, 1985, pp. 223-248; Volpi, 1988, pp. 203-230; Volpi, 1990, pp.3-28; Wood, 2002, 24; y también del mismo Heidegger, GA 3, p. 7.
} 
dice; tiene en cambio que captar lo que el autor quería decir, aun cuando él mismo no fuera plenamente consciente de ello. En este sentido, el abordaje de los textos kantianos, que Heidegger lleva a cabo con frecuencia ${ }^{2}$, no se limita a explicar al lector lo que Kant quería decir, sino que fuerza el sentido original de su pensamiento en una dirección específicamente heideggeriana. Heidegger encuentra siempre algo de sí mismo en los textos que lee y la interpretación de Kant se presenta como “otro más de sus célebres forzamientos”3.

En Phänomenologische Interpretationen von Kants Kritik der reinen Vernunft, Heidegger se introduce impetuosamente en la renombrada Crítica de La Razón Pura con el fin de superar la acepción tradicional de metafísica para poner en evidencia el verdadero sentido del Ser, es decir, el ser como temporalidad en el mundo. Como el filósofo mismo escribe en la introducción de Kant und das Problem der Metaphysik:

Während der Ausarbeitung der WS 1927/28 gehaltenen Vorlesung über 'Kants Kritik der reinen Vernunft' wurde ich auf Schematismuskapitel aufmerksam und erblickte darin einen Zusammenhang zwischen dem Kategorienproblem, d.h. das Seinsproblem der überlieferten Metaphysik und dem Phänomen der Zeit (...). Kants Text wurde eine Zuflucht, bei Kant einen Fürsprecher für die von mir gestellte Seinsfrage zu suchen (XIV) (GA 3, p. 3).

En el presente artículo nuestra manera de proceder estará marcada por la individuación de los puntos del pensamiento de Kant sobre los que Heidegger ha ejercido su forzamiento. Nos concentraremos en forma particular sobre la primera interpretación de la Crítica kantiana que el filósofo dio en las lecciones del semestre de invierno 1927/28. En efecto, es en este curso donde es posible reconocer la primera intervención por parte de Heidegger dirigida a la superación de la metafísica tradicional kantiana y a la construcción de su propia ontología. Insistiremos de manera particular sobre los conceptos de intuición, imaginación y representación que consideramos fundamentales para la formulación de esa ontología. La descripción de este núcleo de conceptos parece, en efecto, no sólo forzar el sentido que Kant les asignaba, sino que además da lugar a ciertas ambigüedades que repercuten en la definición heideggeriana de ontología.

\section{Entre metafísica y ontología}

El punto de partida del análisis de Heidegger consiste en la apropiación filosófica, como ontología fundamental ${ }^{4}$, de la Crítica de la Razón Pura. La Crítica de la Razón Pura, cuyo primer objetivo es captar y aclarar el sentido auténtico de la filosofía, se presenta como un análisis crítico de los fundamentos de la ontología. Según Heidegger, en efecto, “Ontologie (...) ist nichts anderes als Wesen der Philosophie” (GA 25, p. 37). La filosofía, a su vez, es entendida o bien como una investigación de entes específicos, y por lo tanto como una ontología regional (regional Ontologie), o bien como instrumento de análisis del ser en general, es decir, como ontología fundamental (Fundamentalontologie) ${ }^{5}$.

Para Kant, según Heidegger, el problema principal es el de justificar una filosofía que valga como ciencia. La filosofía debe, en efecto, poder demostrar la validez de sus propios fundamentos, en la definición que ella misma da de las condiciones de posibilidad del ser en general y del ser mismo. En términos heideggerianos, de lo que es preontológico y ontológico ${ }^{6}$.

\footnotetext{
Citamos a continuación sólo algunos de los textos más significativos en los que Heidegger se refiere a Kant: GA 1;

GA 2; GA 3; GA 9; GA 21; GA 41.

Véanse: Volpi, 1997, 35; 1996.

Cf. GA 25, § 3.

Cf. GA 25, pp. 37, 38.

Cf. GA 25, p. 39.
}

Studia Heideggeriana, Vol. I, 2020, $2^{\text {a }}$ ed. 
En primer lugar, por consiguiente, la Crítica de la Razón Pura se ocupa para Heidegger del fundamento de la filosofía, entendida como metafísica, en cuanto ciencia válida ${ }^{7}$. Criticando la facultad de juicio, se propone explicar como el ón, ente suprasensible, puede ser captado y descripto. De manera más detallada, ambos filósofos se interrogan sobre la posibilidad de la existencia de juicios sintéticos a priori, capaces de comprender "das vorontologische beziehungsweise ausdrücklich ontologische Seinsverstaendnis des Seienden möglich” (GA 25, pp. 51-52), o sea, el ser sensible según su estructura universal.

En este punto aparece ya el primer forzamiento de Heidegger. En el segundo párrafo, en efecto, el filósofo introduce el concepto de hombre, mientras que Kant en cambio no alude a ninguna forma de subjetividad explícita, ni siquiera a la del "yo pienso". Para Heidegger la ciencia es una condición existencial del ser-ahí (Dasein) humano y no solamente una "manera de conocer" (GA 25, pp. 18-19). El conocimiento no indica aquello que es conocido sino la captación de quién conoce (Greifhalt) (GA 25, pp. 18-19). La palabra “captación” constituye claramente un término clave de la argumentación, ya que indica la relación entre la actitud del sujeto que capta y descubre y el ser. La ciencia describe, según Heidegger, una relación entre “ser-ahí" (existencia) y ente (óv), entre quien descubre y el objeto descubierto, o, si queremos expresarnos desde un punto de vista funcional, entre quien existe y el proceso de objetivación que cumple: "Wir fassen demnach das Erkennen als eine freie Möglichkeit der menschlichen Existenz” (GA 25, pp. 18-9).

El conocimiento del ser, según Heidegger, tiene una raíz ontológica, entendida en sentido existencial y no solamente cognoscitivo. Es efectivamente en la relación entre el sujeto que existe en el mundo y los objetos que de vez en vez descubre, que el sujeto o el ser humano puede justificar frente a sí mismo su existencia. Según Kant, por el contrario, el medio para acceder al conocimiento del ser es el juicio. En uno, la crítica de la metafísica se inicia en la ontología; en el segundo, en el juicio. Los enfoques de los dos filósofos parecen divergir. Ambos tienen como objeto la metafísica; sin embargo le dan, desde un comienzo, funciones distintas: para Kant, es el estudio del ser como conocido; en cambio, para Heidegger, es el estudio del ser-ahí en cuanto sujeto que descubre el ser. El uno está interesado en el desvelamiento del conocimiento, el otro en el de la existencia. El fundamento asumido por Kant es epistemológico, el asumido por Heidegger, ontológico. El primero “descuida”, en su investigación sobre los fundamentos de la ciencia, el problema de cómo se conoce, el segundo de quién conoce.

Esta parece ser la primera y fundamental línea de demarcación entre las maneras de estudiar el ser por parte de los dos filósofos. La ontología de Heidegger se ocuparía de establecer lo que hay, es decir, redactar algo que se parezca a un inventario de lo existente. La metafísica de Kant, en cambio, se ocuparía de establecer qué es lo que hay, es decir, especificar la naturaleza de los objetos incluidos en el inventario ${ }^{8}$. Ambos analizan el propio objeto dando por acertado el propio punto de partida: uno, una epistemología; otro, una ontología válida. Heidegger no se pregunta por el fundamento de lo que es descubierto, Kant no se interroga sobre la posibilidad de la existencia del sujeto juzgante. De todos modos, ambos buscan el fundamento a partir del cual es posible hablar de ciencia y ambos abordan, desde perspectivas distintas, el punto a partir del cual puede originarse una nueva metafísica, entendida como descripción de las condiciones de posibilidad del ser ${ }^{9}$.

\footnotetext{
Cf. GA 25, pp. 12, 40.
}

Acerca de la metáfora del inventario remitimos a Broad, 1923, p. 242.

Naturalmente existe más de una manera de hablar de metafísica y ontología. Existe quien habla de metafísica en un sentido distinto siguiendo su connotación más aristotélica (la metafísica como el estudio de los principios y causas primeras). Véanse por ejemplo los trabajos de Enrico Berti. Existe también quien define a la metafísica como una especulación a priori acerca de aquellos interrogantes que, aún cuando no sea posible resolver a través de la observación y la experimentación, son susceptibles de análisis racional mediante el examen de la estructura de nuestro conocimiento. Citamos en este caso la metafísica descriptiva de P. F. Strawson, 1950, que tanto ha influido en gran parte de la literatura contemporánea de orientación «analítica». Y finalmente concepciones más 
En este sentido se puede estar de acuerdo con la crítica que Berti dirige a la "superación de la metafísica” de Heidegger ${ }^{10}$. Heidegger, en efecto, no parece superar la metafísica tout court; parece, más bien, entrar en una nueva ciencia de las condiciones de posibilidad del ser. Se trata de una ciencia donde está prevista la relatividad del ente respecto al ser-ahí, es decir, los múltiples modos en que los objetos del mundo se dan al hombre y son descubiertos por él. El pensamiento kantiano, en efecto, oscila entre distintas definiciones de metafísica. En primer lugar, la metafísica especial (metaphysica specialis) definida como ciencia que se ocupa de las tres esferas 'Dios, hombre, mundo'. En segundo lugar, la metafísica general (metaphysica generalis) que tendría la finalidad de instituir el presupuesto o el instrumental con el cual desestructurar o replantear un nuevo concepto de metafísica. Y finalmente, la metafísica del fundamento que se preocupa por la aclaración de la raíz oscura del conocimiento. Bajo ninguna de estas tres acepciones Kant analiza la cuestión de la relatividad del ente respecto a un sujeto. A pesar de ello, no puede negarse que tanto la metafísica general como la metafísica del fundamento son explotadas por la actitud destruens y construens de Heidegger para renovar la metafísica tradicional transformándola en una ontología capaz de apoderarse del sentido originario del ser.

La cuestión que planteamos respecto a la nueva metafísica del primer Heidegger es la siguiente: ¿la relatividad del ente respecto al ser-ahí no da lugar a un círculo vicioso que vuelve inútil la ontología misma? El ser-ahí, en efecto, es un ente entre los entes que sin embargo tiene la función de descubrir. El problema es que su descubrir queda siempre a un nivel sensible y no solamente originario, propiamente porque ser-ahí y ente son "arrojados” a la realidad sensible, es decir, al único lugar donde puede tener lugar el proceso del conocer. No obstante, el objetivo de la Crítica es fundar una ontología regional y fundamental capaz de esclarecer las raíces del ser como ente y ser en general. Lo que queremos poner en evidencia, por lo tanto, es la ambigüedad en el nivel existencial entre el ser-ahí que descubre y el ente que es descubierto. Esta ambigüedad, en efecto, se refleja en el proyecto de fundamentación de la ontología heideggeriana, puesto que ni el ser-ahí ni el ente pueden justificar su carácter originario en el proceso de desvelo ${ }^{11}$. En la sección siguiente continuaremos profundizando los detalles de esta ontología y trataremos de llegar a la raíz de la ambigüedad y de la circularidad que hemos encontrado.

\section{La vía de acceso a la ontología}

En la Estética Trascendental Heidegger acepta el presupuesto kantiano según el cual el conocimiento es posible a través de juicios sintéticos a priori. El ente es conocido por intermedio de la estructura sensible e intelectiva del sujeto. Los entes, en efecto, son percibidos

antiinmanentistas y trascendentalistas de la metafísica, de inspiración rosminiana. Citamos aquí: Sciacca, 1950. En cuanto a la ontología, se puede hablar en términos puramente formales (como el estudio de los principios que gobiernan el «ser en cuanto ser», prescindiendo de su manifestación concreta es sectores específicos de la realidad). Tenemos en mente aquí a los filósofos que se apoyan en Husserl; o existe quien, como los filósofos de inspiración heideggeriana o estructuralista, prefiere hablar en clave fenomenológica, en el sentido de análisis de los «modos de darse» el ser a un sujeto o de modo hermenéutico como análisis el poder «constitutivo» de nuestro lenguaje.

10 Cf. Berti, 1985.

11 Estos interrogantes se dirigen claramente al primer Heidegger que comenta el texto kantiano al cual nos referimos aquí. El segundo Heidegger completará su ontología en una analítica existencial que reconocerá la supremacía del ser-aquí respecto del ente. Véase Historical Dictionary of Heidegger's Philosophy, 2000: “Ontology is the science of the being of entities. It inquires into the being of entities in general and tries to ground entities as a whole in the being of a highest entity. This double line of questioning determines the onto-theo-logical constitution of metaphysics" p. 165; "Heidegger's relation to metaphysics is ambiguous. (...) Metaphysics is determined by its onto-theo-logical constitution. As ontology, metaphysics names Beingness as the fundamental quality, which makes an entity what it is and that it is.” (p. 148) 
por la sensibilidad y pensados por el intelecto. Como indicábamos en la sección anterior, el problema principal de este proceso de conocimiento reside en el lugar y la manera en que la síntesis se produce. ¿Quién lleva a cabo la síntesis de lo que la sensibilidad percibe y el intelecto piensa? ¿Cómo se comunican entre sí estas dos funciones tan distintas? En este nudo se encuentra el segundo forzamiento de Heidegger que analizaremos a continuación.

Entre la primera y la segunda edición de la Crítica, Heidegger lamenta el progresivo alejamiento de Kant de la nueva metafísica, la que antes hemos definido como metafísica del fundamento, para acercarse a una acepción más tradicional de molde cartesiano. De hecho, en la segunda edición, Kant quita espacio a la facultad de la intuición y de la imaginación para dar más espacio a la subjetividad abstracta y vacía del Ich denke. En el pasaje de una edición a la otra, el filósofo lleva a cabo, según Heidegger, un verdadero proceso de reubicación de las funciones. Mientras en la primera edición la imaginación era una función de la sensibilidad y a ella pertenecía la tarea de recoger los datos necesarios sobre los cuales la intuición trabaja y el intelecto piensa, en la segunda edición la imaginación es definida como una facultad del intelecto. La tarea de pensar y sintetizar los datos de la sensibilidad no pertenece más a la imaginación productiva, sino a las categorías. Para Heidegger, en la primera edición, la síntesis es confiada a la imaginación; en la segunda, a las categorías. Las raíces del conocimiento para el primer Kant son “sentido, imaginación y apercepción” (A 94 n.) y el intelecto queda relegado como una facultad de importancia secundaria. En la segunda edición, en cambio, las facultades principales del conocimiento son la sensibilidad y el intelecto.

Procedamos ahora según el orden argumentativo seguido por el mismo Heidegger. Todo conocimiento comienza gracias a la intuición que ordena la realidad según los filtros del espacio y del tiempo. La intuición puede ser originaria o derivada. La intuición originaria es, a decir verdad, inescrutable y pertenece a Dios; la misma consiste en una especie de intuición intelectual infinita que comprende el origen de aquello que es intuido. La intuición derivada pertenece, en cambio, a los hombres y es pura o empírica ${ }^{12}$. La primera permite individuar aquellas raíces que puedan justificar la idea de ciencia, a diferencia de la segunda que consiste en la sensación, es decir, en aquel sentimiento creado por la relación entre el sujeto que percibe y el fenómeno percibido, o bien, en el lenguaje de Heidegger, entre captación e intentum. Es por lo tanto en esta función cognoscitiva del sujeto que se encuentra el camino para descubrir el objeto en sí mismo, o sea, la estructura originaria del ente. Es por eso que, en la interpretación de Heidegger, el concepto de intuición posee una función mucho más importante que la que le asigna Kant. Heidegger se ve en parte influido por la bipolaridad de la descripción fenomenológica husserliana del acto, consistente en la correlación entre nóesis y nóema, o sea, entre el sujeto que cumple el acto y el objeto contenido en el acto. La vía de acceso a la estructura de la cosa intuida está siempre abierta y accesible ${ }^{13}$. Para mostrar el ente es suficiente analizar el acto primario dirigido hacia el ente, o sea, la intuición.

En este sentido, la intuición termina siendo, en el análisis heideggeriano, la clave esencial para comprender el punto en el que se origina "la libertad de captación del ser-ahí”. La intuición representa, efectivamente, la función primaria y originaria que describe la relación entre el ser humano que vive y el objeto que es vivido. La intuición es capaz de recibir representaciones, gracias a las afecciones en las que se apoya, y de otorgar sentido al ente que es percibido ${ }^{14}$. Según el análisis de Heidegger el punto de acceso a la estructura originaria del ente, no es el noûs sino la intuición, o sea, la capacidad del sujeto de vivir en el mundo de manera sensible.

Desde este punto de vista es por lo tanto posible insistir en que la via de acceso a la metafísica heideggeriana no es epistemológica, sino ontológica y casi antropológica, ya que para acceder al sentido del ser conocido, es necesario analizar aquello que su maestro llamaría el Erlebnis, lo

\footnotetext{
Cf. GA 25, pp. 85, 101.

Cf. Cassinari, 1994 y Elliott, 2005.

Cf. GA 25, pp. 89-93.
}

Studia Heideggeriana, Vol. I, 2020, $2^{\text {a }}$ ed. 
vivido del sujeto viviente. La intuición se convierte en la principal vía de acceso a la ontología heideggeriana, esto es, se convierte en aquella ciencia capaz de comprender las condiciones de posibilidad del ser, entendido como ser-ahí descubridor o como ente. Veremos a continuación cómo Heidegger define la intuición y como ésta se relaciona con la ontología.

\section{El don de la intuición}

En el estudio de la Analítica de los conceptos, Heidegger define la lógica trascendental como una lógica ontológica, o sea, como "eine solche Untersuchung des Denkens und seiner Elemente, die diese im Hinblick darauf enthüllt, wie sie eine apriorische Erkenntnis der Seinsverfassung des Dienenden möglich machen”(GA 25, p. 170). La ontología, de cierto modo, se funda en la lógica, es decir, en el logos a través del cual puede expresarse ${ }^{15}$. Por lo tanto, para Heidegger la lógica es el instrumento de explicación del ser del ente y puede, por intermedio del pensamiento, reunir, según reglas, los datos recibidos por la sensibilidad. Heidegger, siguiendo la enseñanza de Husserl en las Investigaciones Lógicas $^{16}$, subraya cómo la lógica no es un organon sino el conjunto de los principios que hacen posible la explicación de lo que vivimos ${ }^{17}$. En sentido estricto, la lógica no consiste en la aplicación de estas reglas, sino en los contenidos a partir de los cuales tales reglas se construyen. Ella puede sintetizar lo múltiple apoyándose en la imaginación y la intuición y conceptualizarlo de manera óntica y ontológica es decir, según determinadas categorías de conocimiento, según la categoría del ser en general ${ }^{18}$. En definitiva, la lógica cubre con conceptos positivos el material recogido y crea categorías para ordenarlo.

En el análisis de esta última parte reside el tercer forzamiento del análisis de Heidegger y quizás el más relevante desde el punto de vista de nuestra argumentación. La polémica aquí es particularmente vivaz, porque se pretende fundar una 'metafísica' propia y forzar el contenido expresado en la Analítica de los conceptos de Kant. En esta sección, el principal objeto sometido a examen es el esquematismo trascendental. Se persigue explicar cuál es el lugar en el que se originan los conceptos, qué función ejercitan conjuntamente y de manera coordinada el pensamiento y la intuición y cómo es que se forman las categorías. Las categorías, así como los conceptos, se originan a partir de la idea de síntesis. Pero la síntesis es definida por Heidegger como una operación oscura que en positivo no pertenece ni a la intuición ni al intelecto ${ }^{19}$. Mientras Kant la identifica con la función del juicio y de manera más específica con el acto de la reflexión ${ }^{20}$, Heidegger atribuye la síntesis a la facultad de la imaginación. A la imaginación y no al juicio corresponde la capacidad de sintetizar lo múltiple. En efecto, la imaginación funciona según el filósofo como punto de unión de la multiplicidad de los conceptos sobre los cuales es posible reflexionar sólo una vez que ella ha tenido lugar. Esta, y no el intelecto, parece ser la función que está en la base de la lógica o, si queremos, de la elaboración del conocimiento.

De este modo, distinguiéndose de Kant, Heidegger asigna únicamente a la imaginación la capacidad de síntesis. Heidegger escribe: “Die Synthesis überhaupt ist (...) die bloße Wirkung der Einbildungskraft, einer blinden, obgleich unentbehrlichen Funktion der Seele, ohne die wir überall gar keine Erkenntnis haben würden” (GA 25, p. 276). Esta capacidad reside en el hecho que la imaginación es una particular facultad de la intuición, que está en condiciones de reunir

\footnotetext{
Cf. GA 25, p. 167.

Cf. Hua XIX.

Cf. GA 25, p. 180.

Cf. GA 25, pp. 285-6.

Cf. GA 25, p. 273.

Cf. Ibíd.
}

Studia Heideggeriana, Vol. I, 2020, $2^{\text {a }}$ ed. 
en sí la multiplicidad de objetos que se dan en la forma pura del espacio y del tiempo ${ }^{21}$. Es una especie de intuición pura, destituida de cualquier forma de afección por parte del objeto sensible. La intuición en lo específico es presentada por el filósofo como don con el cual lo múltiple se da, en cuanto objeto, frente al sujeto (Gegen-stand $)^{22}$. La imaginación, entendida por el filósofo como una intuición sin afección, tiene por única función la de reunir la multiplicidad de modo que se forme el contenido del concepto sin que éste sea percibido directamente. Él escribe: "diese Synthesis entspringt rein nur der Einbildungskraft, und diese ist eine 'blinde, obgleich unentbehrliche Funktion der Seele’, die für Möglichkeit der Erkenntnis ursprünglichste Bedingung ist” (GA 25, p. 275). La actividad de la imaginación representa, así, la máxima condición que da origen al descubrimiento del ente por parte del ser.

Tratemos ahora de entender de manera más precisa cómo actúa la imaginación y si es posible o no trazar una línea de demarcación entre la identidad del ser-ahí que formula la pregunta y la del ente descubierto. Es en esta función donde se encuentra el punto de origen de aquella metafísica circular y ambigua a la cual hemos aludido en los párrafos anteriores. Si la imaginación es en efecto el lugar donde tiene origen el proceso de descubrimiento del ente por parte del ser y si ella opera sin necesidad de afección, su pureza y carácter originario permanecen intactos. La circularidad ontológica entre ente y ser-ahí resulta interrumpida por este don que originalmente pertenece al ser-ahí gracias a su capacidad de intuir y, aún antes, de imaginar. Veamos entonces más de cerca cómo opera.

Heidegger confiere a la imaginación la tarea de unificar la multiplicidad pura de los entes que son captados, ante todo, en cuanto temporalidad y por lo tanto privados de su contenido. Ellos son, en substancia, aquellos conceptos vacíos que hacen que sea posible reflexionar sobre los objetos y formular conceptos con contenido. "Die reinen Verstandesbegriffe GA 25, p. 313). La imaginación puede llevar a cabo esta síntesis a través de tres diversas actividades, a saber, la aprehensión, la reproducción y el reconocimiento. Estas tres actividades marcan las tres caras del tiempo: síntesis de lo que es presente, pasado y futuro. Estas formas, siendo síntesis del tiempo, son también definidas por Heidegger como formas del tiempo. La aprehensión, en efecto, considera al objeto tal y como aparece en el pasado, la reproducción se ocupa de sintetizarlo en sus actualizaciones presentes y el reconocimiento se preocupa de prever su morfología en el futuro ${ }^{23}$. Para que estas operaciones sean posibles es absolutamente indispensable la presencia de un sujeto al cual los objetos se dan anticipadamente dentro de un determinado horizonte temporal.

Según Heidegger, a diferencia de Kant, las categorías no pueden ser lugar de síntesis ni lugar de producción. Es la función representativa, y no las categorías, el lugar donde tiene origen el conocimiento y ella misma es situada por Heidegger dentro de la facultad general de la intuición. La imaginación, como facultad productiva y representativa, media entre intuición e intelecto, desarrollando así su función primaria de actividad representativa ${ }^{24}$. En la capacidad representativa de la imaginación reside la verdadera capacidad de síntesis de lo múltiple y de descubrimiento del ente. El verdadero fundamento del conocimiento ontológico está originalmente constituido por el representar (Vorstellung, o sea: stellen vor). Mediante este conocimiento el ente se vuelve simplemente presente (vorhanden) y dotado de una estabilidad propia en el fluir del tiempo. La mera presencia constituye la modalidad ontológica con la cual el objeto es en su estar frente al sujeto. El ente imaginado, en efecto, representa su prae ens, es decir, el ser ya constituido como ente en su ser objeto ${ }^{25}$.

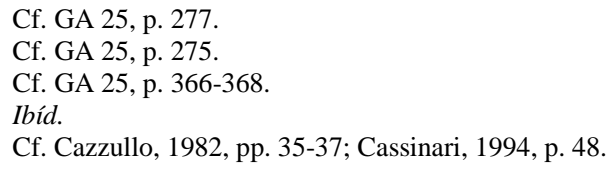


La representación, como función de la imaginación, es por lo tanto central, ya que constituye el instrumento para adquirir verdad. El problema es que la verdad es adecuación al objeto y lo mismo sucede con la representación. Y, más en general, la imaginación y la intuición son facultades que "responden" y se adecuan a la necesidad de conocimiento del ente. En este sentido, la intuición como género de la representación no puede ser originaria o absoluta, sino sensible en cuanto duplicado del objeto representado y don espontáneo derivado de la presencia de la relación sujeto-objeto. La función representativa y por lo tanto cognoscitiva, hecha explícita por la intuición en general y por la imaginación en particular, lleva a interpretar el ser originario de lo intuido (y por lo tanto también de lo imaginado) como adequatio rei.

El don de la intuición pues, a diferencia de lo dicho respecto de la imaginación, no puede ser completamente puro y originario, puesto que se presenta como una especie de respuesta respecto de lo real. Si la representación es realmente la función propia tanto de la intuición como de la imaginación y si ella es una adequatio rei, la intuición y la imaginación no pueden explicar el descubrimiento originario del ser porque también ellas se adecuan al ente. La "respuesta” de la representación muestra las categorías y, aún más, los modos del tiempo (aprehensión, reproducción y reconocimiento) que de ella se generan, no como primarios sino como reacciones que responden a la realidad del objeto.

\section{Conclusión}

En resumen, podemos reconocer que del primer análisis heideggeriano de la Crítica de Kant emerge la ambición de fundar una metafísica capaz de superar los límites epistemológicos y, sobre todo, ontológicos de la metafísica tradicional kantiana. Sin embargo, en este análisis los límites son solamente fijados y no resueltos. La cuestión acerca del Ser-ahí y acerca de la manera en la que se produce la síntesis con respecto al ente, abre una circularidad que permanece ambigua. Por un lado, la facultad de la imaginación parece justificar la pureza del Ser-ahí respecto al ente, por el otro, sin embargo, la definición de representación lleva a entender el Serahí como una respuesta al ente. Una vez más, entre Ser-ahí y ente se genera una continuidad que vuelve difícil establecer los límites entre aquel que se pregunta por el ser y el ente que se conforma a lo que se recibe. La intuición, pues, es la función del representar, pero si el representar es un adecuarse al objeto, la intuición no puede ser una 'pura' aprehensión del objeto. Ambas funciones dan origen a una especie de círculo vicioso, porque la intuición es condición de posibilidad del conocimiento de los objetos, debido a la forma del tiempo, pero al mismo tiempo necesita de la existencia de los objetos para poderlos recibir. El ser recibido puramente es consecuencia de una "reacción" de adecuación frente a lo que se tiene enfrente. La ontología, o si queremos la metafísica del fundamento, así delineada no es en este sentido una 'pura' metafísica, una apropiación del sentido fundamental del ser en general, como Heidegger había afirmado al inicio de las lecciones, sino que se, por así decirlo, queda circularmente encerrada dentro de esta relación de don y reacción establecida entre sujeto y objeto o, si se quiere, entre sujeto que intuye y objeto representado. En fin, Heidegger al forzar en distintos puntos la Critica kantiana, termina por fundar una ontología que no está aún en condiciones de mostrar el ser como región específica o general (ontología regional o fundamental) y consecuentemente tampoco de justificar la filosofía como ciencia capaz de mostrar la validez de estas formas del ser. Esta es, según nuestro análisis, una consecuencia de la definición todavía ambigua que el filósofo elabora alrededor de la relación existente entre intuición, imaginación y representación. 


\section{Referencias}

BERTI, Enrico (1985), “Überwindung della metafisica?”, en La metafisica e il problema del suo superamento, Gregoriana, Padova.

BROAD, Charlie Dunbar (1923), Scientific Thought, Routledge, Londres

CASSINARI, Flavio (1994) Definizione e rappresentazione. Antropologia e metafisica nell'interpretazione heideggeriana di Kant, Guerini Studio, Milano.

CAZZULLO, Aldo (1982), Heidegger: immagine e rappresentazione, Unicopli, Milano.

ELLIOTT, Brian (2005), Phenomenology and Imagination in Husserl and Heidegger, Routledge, London.

DENKER, Alfred (2000) Historical Dictionary of Heidegger's Philosophy, the Scarecrow Press, Lanham, Maryland and London.

GADAMER, Hans-Georg (1983), Heideggers Wege. Studien zum Spätwerk, Mohr, Tübingen.

HEIDEGGER, Martin, GA 1, Das Realitätsproblem in der modernen Philosophie, en Frühe Schriften, ed. F. W. v. Herrmann, Frankfurt a.M., Klostermann, 1978.

HEIDEGGER, Martin, GA 2, Sein und Zeit, ed. F-W. von Herrmann, Niemeyer, Tübingen, 1977.

HEIDEGGER, Martin, GA 3, Kant und das Problem der Metaphysik, ed. F.-W. v. Herrmann, Frankfurt a.M., Klostermann,1973.

HEIDEGGER, Martin, GA 9, Kants These über da Sein, en Wegmarken, ed. F.-W. v. Herrmann, Frankfurt a.M., Klostermann 1a ed., 1976.

HEIDEGGER, Martin, GA 9, Was ist Metaphysik?, en Wegmarken, ed. F.-W. v. Herrmann, Frankfurt a.M., Klostermann, 1976.

HEIDEGGER, Martin, GA 21, E. Cassirer, Philosophie der symbolischen Formen. 2. Teil: Das mysthische Denken; Logik. Die Frage der Wahrheit, ed. Walter Biemel, Frankfurt a.M., Klostermann, 1976.

HEIDEGGER, Martin, GA 25, Phänomenologische Interpretation von Kants Kritik der reinen Vernunft, ed. F.W. von Herrmann, Frankfurt a.M., Klostermann, 1977.

HEIDEGGER, Martin, GA 41, Die Frage nach dem Ding. Zu Kants Lehre von den transzendentalen Grundsätzen, ed. Petra Jaeger, Frankfurt a.M., Klostermann, 1984.

HUSSERL, Edmund (1975), Logische Untersuchungen. Prolegomena zur reinen Logik, ed. Holstein, Martinus Nijhoff, The Hague.

SCIACCA, Michele Federico (1950), Filosofia e metafisica, Morcelliana, Brescia.

STRAWSON, Peter Frederick (1959), Individuals. An Essay in Descriptive Metaphysics, Methuen, Londres.

VOLPI, Franco (1984), Heidegger e Aristotele, Daphne, Padova.

VOLPI, Franco (1985), “Heidegger e i Greci”, en Ars Majeutica. Studi in onore di Giuseppe Faggin, Neri Pozza, Vicenza, pp. 223-248;

VOLPI, Franco (1988), “L'approccio fenomenologico alla storia della filosofia del primo Heidegger", en Aut Aut, no. 223-224, pp. 203-230

VOLPI, Franco (1990), "La riabilitazione della dynamis e della enérgheia in Heidegger", en Aquinas, no. 33, pp. 3-28;

VOLPI, Franco (1996), La question du logos chez le Jeune Heidegger, en ed. J. F. Courtine, Heidegger 1919-1929. De l'herméneutique de la facticité á la mataphysique du Dasein, Paris.

VOLPI, Franco (1997), Guida ad Heidegger, Laterza, Roma-Bari.

WOOD, David (2002), Thinking after Heidegger, Stephen Mulhall, New College, Oxford. 